\title{
Pterostilbene inhibits reactive oxygen species production and apoptosis in primary spinal cord neurons by activating autophagy via the mechanistic target of rapamycin signaling pathway
}

\author{
JING-LAN HE, XIAO-HUI DONG, ZONG-HU LI, XIAO-YING WANG, ZHI-AN FU and NA SHEN \\ Department of Orthopedic Surgery, The Affiliated Hospital of \\ Hebei University of Engineering, Handan, Hebei 056002, P.R. China
}

Received August 25, 2017; Accepted November 13, 2017

DOI: $10.3892 / \mathrm{mmr} .2018 .8412$

\begin{abstract}
Autophagy is an important self-adaptive mechanism that is involved in inhibiting reactive oxygen species (ROS) in spinal cord neurons. Pterostilbene, a natural plant extract, has been demonstrated to possess antioxidant effects; however, it has not yet been investigated whether pterostilbene could activate autophagy and protect spinal cord neurons from oxidative stress. In the present study, primary spinal cord neurons of Sprague Dawley rats were cultured. Cell counting kit-8 analysis was used to detect cytotoxicity of pterostilbene. Cells were treated with various doses of pterostilbene for 24 and $48 \mathrm{~h}$, respectively, and $\mathrm{H}_{2} \mathrm{O}_{2}$ was used to induce ROS production. Western blot analysis was performed to assess the protein expression of microtubule-associated protein 1 light chain 3 (LC3)-II, Beclin-1, p62, p-p70S6K and p-mechanistic target of rapamycin (mTOR). Furthermore, the green fluorescent protein (GFP)-LC3 assay was used to detect the level of autophagy level and activation mechanism. 2',7'-Dichlorofluorescin diacetate and MitoSOX Red staining were used to detect ROS production, and Terminal deoxynucleotidyl-transferase-mediated dUTP nick end labelling assay was used to analyze apoptosis percentage. ATG5 small interfering (si)RNA transfection was used to analyze the involvement of autophagy. A dose-dependent increase in the expression of LC3-II and Beclin-1, as well as the p62 decline, were observed in the pterostilbene-treated neurons; however, p-p70S6K and p-mTOR expression was inhibited by pterostilbene. Pterostilbene increased the expression of LC3-II in $\mathrm{H}_{2} \mathrm{O}_{2}$-treated cells, and GFP-LC3 analysis demonstrated an increased number of autophagosomes. Furthermore, pterostilbene significantly inhibited the ROS production and apoptosis induced by $\mathrm{H}_{2} \mathrm{O}_{2}$; however, ATG5 siRNA transfection
\end{abstract}

Correspondence to: Dr Na Shen, Department of Orthopedic Surgery, The Affiliated Hospital of Hebei University of Engineering, 81 CongTai Road, Handan, Hebei 056002, P.R. China

E-mail: shen_na2017@163.com

Key words: spinal cord neurons, pterostilbene, autophagy, oxidative stress significantly reversed the protection of pterostilbene. These results indicate that pterostilbene may inhibit the ROS production and apoptosis in spinal cord neurons by activating autophagy via the mTOR signaling pathway.

\section{Introduction}

The mortality and paralysis rate of spinal cord injury (SCI) remains remarkably high, but an effective treatment is still required and SCI pathology is still unclear (1). SCI pathology includes initial injury and secondary injury of spinal cord neurons. Secondary injury after SCI is considered to be an important mechanism of SCI progress, including spinal cord neurons inflammation, apoptosis, death, demyelination, and axonal degeneration (2). Furthermore, secondary injury is considered to be a key cause of clinical symptoms of SCI, therefore treatment and prevention of secondary injury to improve the prognosis of SCI is of great significance (1). Reactive oxygen species (ROS) and oxidative stress have been thought to be the main cause for the secondary injury with the apoptosis and death of spinal cord neurons, leading to the SCI progression (3). Taken together, alleviation of ROS and oxidative stress in spinal cord neuron might be an alternative method to treat the SCI (4). In this paper, the primary spinal cord neurons were exposed to $\mathrm{H}_{2} \mathrm{O}_{2}$ to mimic the oxidative stress confronted by the cells after SCI.

Pterostilbene is a natural plant antitoxin that exists in a variety of plants, including blueberries and grapes (5). Importantly, pterostilbene is an analog of resveratrol which has been acknowledged as an anti-oxidative stress and anti-tumor drug (5). Pterostilbene has a variety of pharmacological effects, including anticancer, anti-inflammatory response, anti-oxidative stress and anti-diabetes. Compared with the resveratrol, pterostilbene has a characteristic of better oral absorption and metabolic property (5). In the nervous system, pterostilbene can protect central neuronal cells from the damage caused by ischemia and hypoxia (6). Oral pterostilbene can relieve the injury of neuronal cells caused by ischemia-reperfusion (7); however, the effect of pterostilbene on spinal neuron cells has not been reported.

Autophagy, a self-protecting mechanism, maintains cellular homeostasis by fusing with lysosomes to degrade 
malformed proteins and damaged organelles $(8,9)$. Under stress conditions, moderately activated autophagy is important for the survival and adaptation of spinal cord neurons (10). It has been reported that autophagy could prevent spinal cord neurons from apoptosis induced by oxidative stress (11); however, the effect of autophagy on ROS production remains unclear in spinal cord neuron. Given the role of autophagy in ROS production in other cells, we hypothesize that autophagy could inhibit ROS production and apoptosis in spinal cord neuron. Furthermore, it has been reported that pterostilbene could activate autophagy in human oral cancer cells and vascular endothelial cells $(12,13)$; however no related reports have been reported in spinal cord neurons.

In this study, primary spinal cord neurons were cultured and treated with pterostilbene, and the effects of pterostilbene on neuronal autophagy, ROS production and apoptosis, as well as the involvement of autophagy in the ROS production and apoptosis, were investigated.

\section{Materials and methods}

The animal protocols in this study were approved by the Animal Care and Use Committee of Affiliated Hospital of Hebei University of Engineering (Handan, China). The animal experiments were performed according to Guidance Suggestions for the Care and Use of Laboratory Animals, which were formulated by the Ministry of Science and Technology of China.

Reagents and antibodies. Primary antibodies of neuron specific enolase (NSE), light chain 3 (LC3)b, Beclin-1, p62, ATG5 and $\beta$-actin were purchased from Abcam (Cambridge, MA, USA); 2',7'-dichlorofluorescin diacetate (DCFDA) Staining kit, western blotting, secondary antibody labeled with Alexa Fluor-555, 4',6-diamidino-2-phenylindole (DAPI) and protein extraction kits were bought from Beyotime Institute of Biotechnology (Haimen, China); Cell culture reagents, including Neurobasal-A, fetal bovine serum (FBS) and $0.25 \%$ trypsin, were obtained from Thermo; Cell Counting Kit-8 (CCK-8) was purchased from Dojindo Molecular Technologies, Inc. (Kumamoto, Japan); MitoSOX Red Staining Kit was purchased from Invitrogen (Thermo Fisher Scientific, Inc., Waltham, MA, USA).

Culture of primary spinal cord neurons. Primary spinal cord neurons culture was carried out according to a published literature (14). Briefly, spinal cord tissues were isolated from embryos of E16 Sprague-Dawley (SD) rats under a microscopy, and spinal meninges and dorsal root ganglias were separated from spinal cords under aseptic conditions. After washing with PBS three times, the spinal cord was digested with $0.25 \%$ trypsin at $37^{\circ} \mathrm{C}$ for $30 \mathrm{~min}$. After digestion, cell suspension was filtered through a 200 mesh sieve. The filtered cells were seeded on plates with Neurobasal-A medium (Thermo Fisher Scientific, Inc.), which was added with $2 \% \mathrm{~B}-27,2 \mathrm{mM}$ glutamine, $1 \mu \mathrm{M}$ cytarabine and $50 \mathrm{ng} / \mathrm{ml}$ nerve growth factor (R\&D Systems, Inc., Minneapolis, MN, USA).

Experimental design. When analyzing pterostilbene cytotoxicity, spinal cord neurons were treated with 1, 5, 10, 20, 40,
60 and $80 \mu \mathrm{M}$ pterostilbene for 24 and $48 \mathrm{~h}$. When analyzing effects of pterostilbene on autophagy in normal spinal cord neurons, neurons were treated with $1,5,10$ and $20 \mu \mathrm{M}$ pterostilbene for $24 \mathrm{~h}$. In order to investigate whether pterostilbene could activate autophagy and regulate ROS production in the neurons under oxidative stress, cells were divided into 4 groups: Control, pterostilbene, $\mathrm{H}_{2} \mathrm{O}_{2}$, pterostilbene $+\mathrm{H}_{2} \mathrm{O}_{2} .10 \mu \mathrm{M}$ $\mathrm{H}_{2} \mathrm{O}_{2}$ were added $1 \mathrm{~h}$ prior to pterostilbene with $24 \mathrm{~h}$ treatment. In order to investigate the involvement in of autophagy in the regulation of ROS production, cells were divided into 4 groups: Control, pterostilbene $+\mathrm{H}_{2} \mathrm{O}_{2}$, pterostilbene $+\mathrm{H}_{2} \mathrm{O}_{2}+$ ATG5 siRNA, Control siRNA. In order to investigate the involvement of autophagy in the regulation of apoptosis percentage, cells were divided into 6 groups: Control, $\mathrm{H}_{2} \mathrm{O}_{2}$, pterostilbene, pterostilbene $+\mathrm{H}_{2} \mathrm{O}_{2}$, pterostilbene $+\mathrm{H}_{2} \mathrm{O}_{2}+$ ATG5 siRNA, Control siRNA.

Cell immunofluorescence. Primary spinal cord neurons were fixed with $4 \%$ paraformaldehyde in 24-well plates for $10 \mathrm{~min}$ and treated with $0.2 \%$ Triton $\mathrm{X}-100$ for $15 \mathrm{~min}$. Then cells were incubated with $5 \%$ goat serum for $30 \mathrm{~min}$ and NSE primary antibody (1:100) overnight at $4^{\circ} \mathrm{C}$. After washing, cells were incubated with Alexa Fluor-555-labeled secondary antibody for $2 \mathrm{~h}$ at room temperature and stained with DAPI (4',6-diamidino-2-phenylindole) for $5 \mathrm{~min}$. Finally, neurons were observed under fluorescence microscopy (Olympus Corporation, Tokyo, Japan).

CCK-8 analysis. A total of 5,000 cells were cultured in each well of 96-wells and treated as the experimental design described. At 24 and $48 \mathrm{~h}$ after treatment, the previous medium was replaced with $100 \mu \mathrm{l}$ fresh medium, and $10 \mu \mathrm{l} \mathrm{CCK}-8$ reagent was added to each well. Then, neurons were incubated in a $37^{\circ} \mathrm{C}$ incubator for $1 \mathrm{~h}$, and OD value was obtained in a microplate reader at $450 \mathrm{~nm}$. The results were normalized by the absorbance of control cells.

Western blotting. Total protein was extracted from neurons by using RIPA contained 1\% PMSF. A bicinchoninic acid (BCA) method was used to determine protein concentration. Then the protein was separated by SDS-PAGE and transferred to Polyvinylidene Fluoride (PVDF) membranes (Bio-Rad Laboratories, Inc., Hercules, CA, USA). The membranes were incubated with $5 \%$ milk for $2 \mathrm{~h}$ and then incubated with LC 3b, Beclin-1, p62 and ATG5 primary antibody (1:1,000 dilution) overnight at $4^{\circ} \mathrm{C}$. After washing three times with TBST, the membranes were incubated secondary antibody labeled with horseradish peroxidase at $37^{\circ} \mathrm{C}$ for $2 \mathrm{~h}$ and exposed to an enhanced chemiluminescence detection system (PerkinElmer, Inc., Waltham, MA, USA) with ECL plus regent (Thermo Fisher Scientific, Inc.). Semi-quantitative analysis of protein bands was performed using an AlphaEaseFC 4.0 software.

Green fluorescent protein (GFP)-LC3 transfection. Spinal cord neurons were cultured on a glass bottom dish with $2 \mathrm{ml}$ medium overnight. When neurons were approximately 50-70\% confluent, cells were transfected with GFP-LC3 adenovirus with 100 multiplicity of infection (MOI) and $10^{10} / \mathrm{ml}$ titer. The adenovirus was purchased from Han Heng Biology (Shanghai, China). The volume and number of virus were $20 \mu \mathrm{l}$ and 
$2 \times 10^{8}$ respectively, which was calculated according to the $100 \mathrm{MOI}$ and $1-2 \times 10^{6}$ cells in each six-well plate. According to manufacturer's protocol, the adenovirus was diluted with $1 \mathrm{ml}$ serum-free medium, and cells were cultured with the $1 \mathrm{ml}$ medium and incubated in a $37^{\circ} \mathrm{C}$ incubator for $2 \mathrm{~h}$. Then, the serum-free medium was replaced with $2 \mathrm{ml}$ normal medium. After culture overnight, cells were observed under fluorescence microscopy to detect transfection efficiency. After treatments described in the experimental design, autophagosomes were observed using a laser confocal microscopy (Leica TCS SP8; Leica Microsystems GmbH, Wetzlar, Germany).

DCFDA staining. DCFDA is a classical method for analyzing total intracellular ROS levels. Neurons were treated with the above-mentioned treatments and incubated with $10 \mu \mathrm{M}$ DCFDA dye in a dish with glass bottom at $37^{\circ} \mathrm{C}$ for $20 \mathrm{~min}$. The cells were observed under confocal microscopy or digested with $0.25 \%$ trypsin followed by semi-quantitative analysis of fluorescence intensity using a flow cytometer (FACSCaliber; BD Biosciences, Heidelberg, Germany) at excitation wavelength of $488 \mathrm{~nm}$ and emission wavelength of $525 \mathrm{~nm}$.

MitoSOX red staining. MitoSOX Red is a new type staining for detecting mitochondrial ROS, which emits red fluorescence when oxidized by mitochondrial superoxide anion $\left(\mathrm{O}^{2-}\right) .50 \mu \mathrm{g}$ MitoSOX Red solution was dissolved in $13 \mu 1$ DMSO to prepare a $5 \mathrm{mM}$ stock solution. Spinal cord neurons were treated with $5 \mu \mathrm{M}$ MitoSOX Red solution at $37^{\circ} \mathrm{C}$ for $15 \mathrm{~min}$, washed with PBS and replaced with fresh Neurobasal-A medium. Finally, cells were observed under confocal microscopy (Leica TCS SP8; Leica Microsystems GmbH).

ATG5 siNRA transfection. ATG5 siRNA sequence was designed based on a previous reference (19), which was shown in Table I and synthesized by Gemma Co., Ltd. (Shanghai, China). Neurons number was counted after digestion, and $2 \times 10^{5}$ cells were added to each well in a 6 -well plate and cultured overnight. Transfection was performed using Lipofectamine ${ }^{\circledR} 2000$ (Invitrogen; Thermo Fisher Scientific, Inc.) according to Invitrogen's protocol. Western blotting was used to analyze the success of silence. At $48 \mathrm{~h}$ after transfection, cells were treated according to the experimental design. Non-specific siRNA was used as a control RNA.

TUNEL staining. Spinal cord neurons were fixed with 4\% paraformaldehyde for $30 \mathrm{~min}$ and incubated with $0.1 \%$ Triton X-100 for 2 min, followed by the incubation with in situ cell death detection kit (Roche, Hertfordshire, UK) for $60 \mathrm{~min}$. Neurons treated without the terminal transfer were used as negative controls; Neurons treated with $2 \mathrm{U} / \mathrm{ml}$ DNase were used as positive controls. Then, cells were stained with DPAI for $5 \mathrm{~min}$ and observed under fluorescence microscopy. Apoptosis percentage was calculated according to the ratio of green nuclei to blue nuclei in 6 fields (x200) each group.

Statistical analysis. The differences between different groups were detected using one-way ANOVA analysis with SPSS15 software (SPSS, Inc., Chicago, IL, USA). If the difference is statistically significant, a LSD method was used to analyze
Table I. Sequence of ATG5 siRNA.

\begin{tabular}{lll}
\hline Primer & Direction & \multicolumn{1}{c}{ Sequence 5'-3' } \\
\hline Atg5 & Sense & GGCCUUUCAUUCAGAAGCUTT \\
& Antisense & AGCUUCUGAAUGAAAGGCCTT \\
Negative & Sense & UUCUCCGAACGUGUCACGUTT \\
Control & Antisense & ACGUGACACGUUCGGAGAATT \\
\hline
\end{tabular}

siRNA, small interfering RNA.

the differences between two groups. $\mathrm{P}<0.05$ was considered to indicate a statistically significant difference.

\section{Results}

Identification of primary spinal cord neurons and the effect of pterostilbene on cell viability. Under a phase-contrast microscopy, primary spinal cord neurons with long and spindle morphology were observed. Typical cells had long neurites on both sides. Some neurites showed bifurcation and intertwined into a network (Fig. 1A). NSE immunofluorescence showed that neuronal cell cytoplasm was rich in red, demonstrating a high expression of NSE protein (Fig. 1A).

In order to investigate the cytotoxicity of pterostilbene on spinal cord neurons, CCK- 8 test was used to analyze the viability of neurons treated with $0-80 \mu \mathrm{M}$ pterostilbene for 24 and $48 \mathrm{~h}$. After 24 and $48 \mathrm{~h}$ treatment, 40, 60 and $80 \mu \mathrm{M}$ pterostilbene significantly inhibited the viability of neuronal cells ( $\mathrm{P}<0.05$, Fig. 1B); however, 1, 5, 10 and $20 \mu \mathrm{M}$ pterostilbene had no influences on the cell viability (Fig. 1B). Therefore, $20 \mu \mathrm{M}$ pterostilbene with $24 \mathrm{~h}$ treatment was used in the following experiments.

Pterostilbene activated autophagy and inhibited mechanistic target of rapamycin (mTOR) pathway in spinal cord neurons. Western blotting was used to analyze the expression levels of LC3-II, Beclin-1 and p62 in spinal cord neurons. A dose-dependent increase in LC3-II/ $\beta$-actin and Beclin- $1 /$ $\beta$-actin expression was observed in pterostilbene-treated cells $(\mathrm{P}<0.05$, Fig. 2A-C). Because p62 forms protein aggregate degraded by autophagy, it was reasonable the p62 expression was decreased gradually by the pterostilbene in a dose-dependent manner, demonstrating a stimulatory role of pterostilbene on autophagic flux in spinal cord neurons $(\mathrm{P}<0.05$, Fig. 2A and $\mathrm{D})$.

mTOR pathway, a classic signaling pathway involving in the autophagic activation, was investigated by Western blotting. As shown in Fig. 2E and F, pterostilbene significantly inhibited the expression of p-p70S6K/p70S6K and p-mTOR/mTOR in spinal cord neurons $(\mathrm{P}<0.05)$.

Pterostilbene activated autophagy in spinal cord neurons treated with $\mathrm{H}_{2} \mathrm{O}_{2}$. In order to further investigate whether pterostilbene could activate autophagy in spinal cord neurons under oxidative stress, cells were treated with $10 \mu \mathrm{M} \mathrm{H}_{2} \mathrm{O}_{2} 1 \mathrm{~h}$ prior to pterostilbene. Pterostilbene significantly increased the 
A
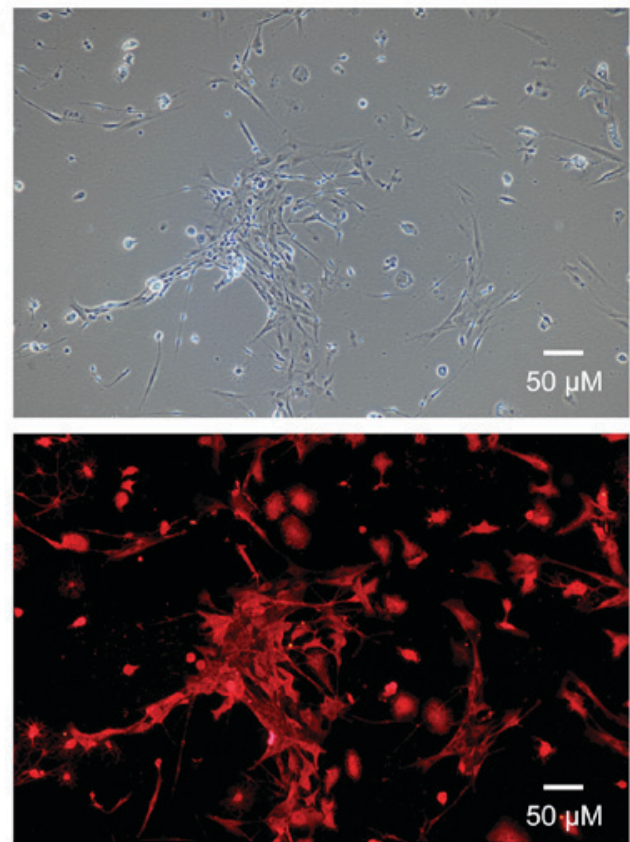

B
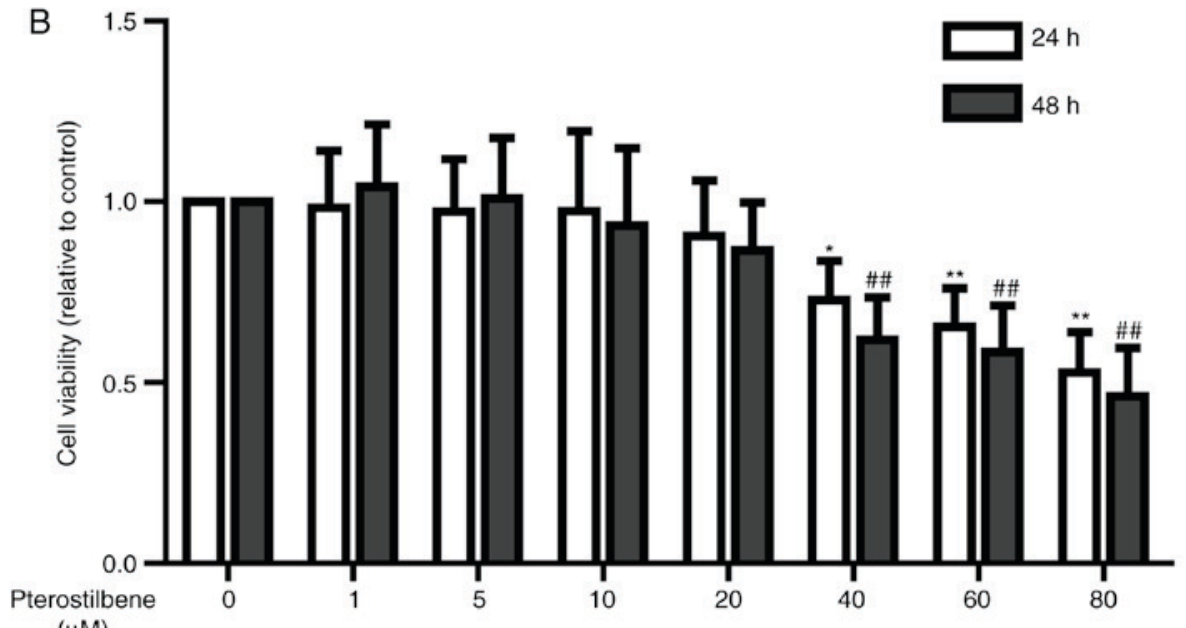
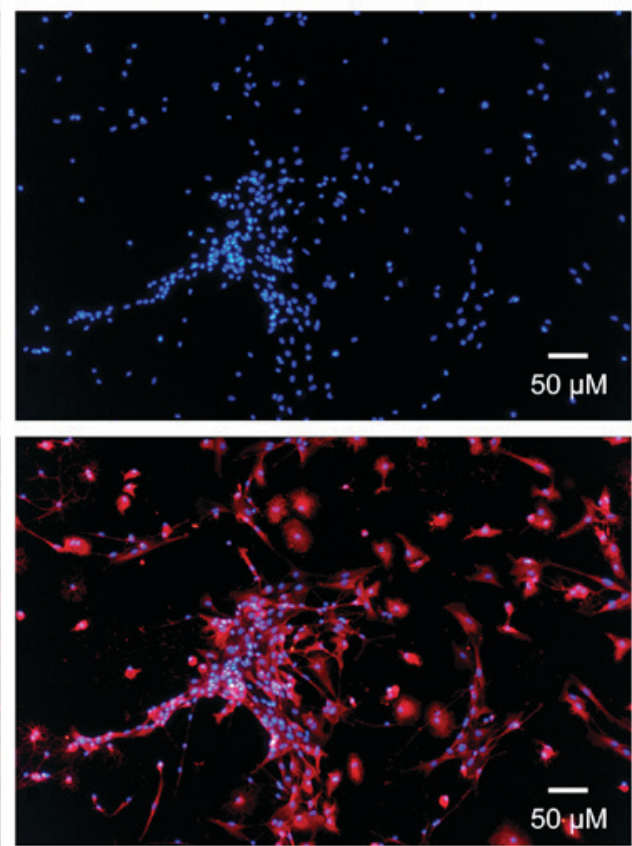

$50 \cdot \mu \mathrm{M}$

$(\mu \mathrm{M})$

Figure 1. The culture of primary rat spinal cord neurons and the effect of pterostilbene on the vitality of primary spinal cord neurons. (A) Primary spinal cord neurons culture and NSE immunocytochemistry. (B) Cell viability after pterostilbene treatment for 24 or $48 \mathrm{~h},{ }^{*} \mathrm{P}<0.05,{ }^{* * *} \mathrm{P}<0.01,{ }^{\# \#} \mathrm{P}<0.01$, compared with control group respectively, $\mathrm{n}=6$. NSE, neuron specific enolase.

level of LC3-II/ $\beta$-actin in the $\mathrm{H}_{2} \mathrm{O}_{2}$-treated spinal cord neurons $(\mathrm{P}<0.05$, Fig. 3A and B). GFP-LC3 assay showed that pterostilbene significantly enhanced the number of LC3-positive green dots in one cell and the number of LC3-positive cells (Fig. 3C). Furthermore, pterostilbene combined with $\mathrm{H}_{2} \mathrm{O}_{2}$ also increased the number of LC3-positive cells compared with $\mathrm{H}_{2} \mathrm{O}_{2}$ alone (Fig. 3C), indicating that pterostilbene could activate autophagy in the spinal cord neurons under oxidative stress.

Pterostilbene inhibits ROS production in spinal cord neurons treated with $\mathrm{H}_{2} \mathrm{O}_{2}$. DCFDA and MitoSOX staining were used to analyze the total ROS and mitochondrial ROS production in spinal cord neurons. It was not surprising that $10 \mu \mathrm{M} \mathrm{H}_{2} \mathrm{O}_{2}$ significantly increased fluorescence intensity of DCFDA and MitoSOX in spinal cord neurons; however, pterostilbene significantly attenuated the increase in ROS production $(\mathrm{P}<0.05$, Fig. 4), suggesting that pterostilbene could inhibited the intracellular ROS production induced by $\mathrm{H}_{2} \mathrm{O}_{2}$.
The involvement of autophagy in the inhibitory effect of pterostilbene on ROS production in spinal cord neurons. ATG5, a key participator of the autophagic process, was silenced by ATG5 siRNA transfection to explore the involvement of autophagy in the inhibitory effect of pterostilbene on ROS production. $48 \mathrm{~h}$ after ATG5 siRNA transfection, the ATG5 expression was significantly inhibited ( $\mathrm{P}<0.05$, Fig. $5 \mathrm{~A}$ and $\mathrm{C})$. Western blotting showed that ATG5 siRNA significantly inhibited the expression of LC3-II/ $\beta$-actin, demonstrating the decline of autophagy $(\mathrm{P}<0.05$, Fig. $5 \mathrm{~B}$ and D). DCFDA and MitoSOX staining showed that ATG5 siRNA significantly increased the ROS production in cells treated with pterostilbene combined with $\mathrm{H}_{2} \mathrm{O}_{2}$, suggesting that inhibition of autophagy reversed the protective effect of pterostilbene against the ROS production in spinal cord neurons (Fig. 5E and F).

Effects of pterostilbene and autophagy on apoptosis of spinal cord neurons under oxidative stress. Since apoptosis has been 
A
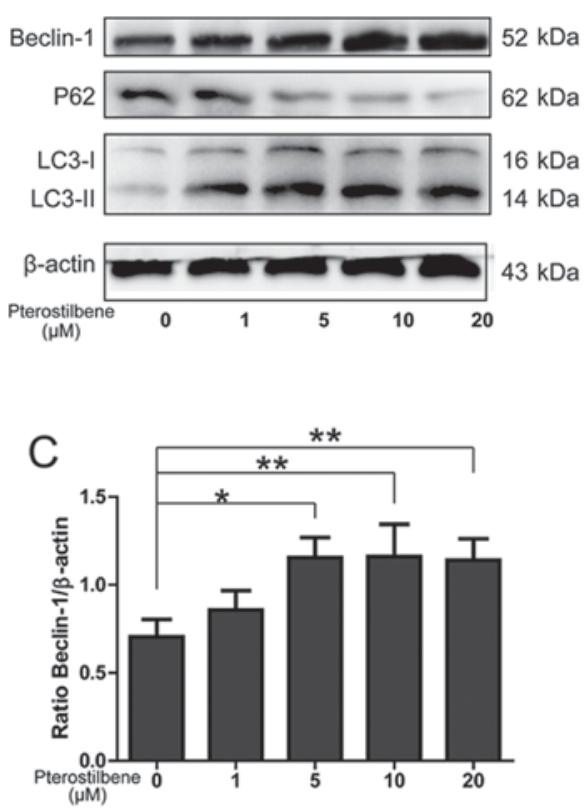

E

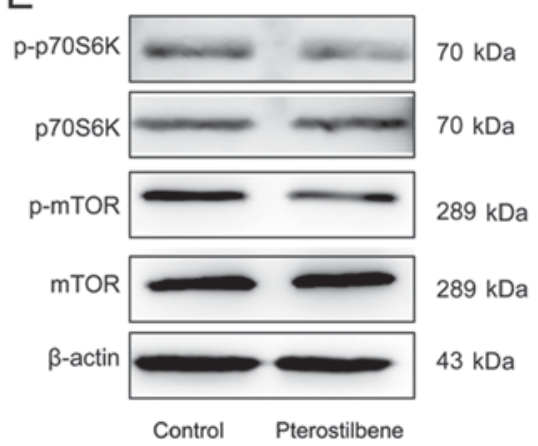

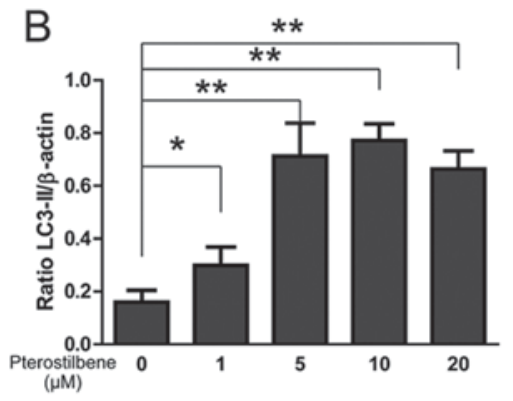
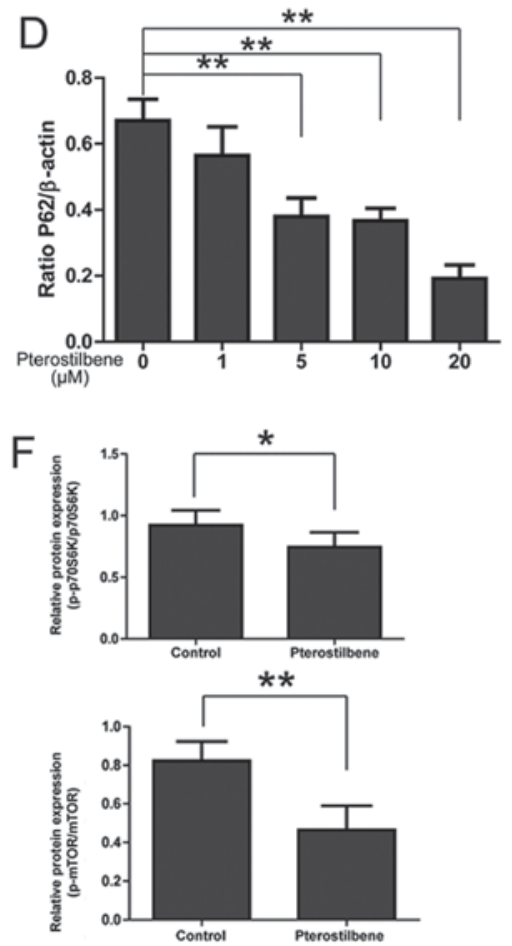

Figure 2. The effect of pterostilbene on the expression of LC3, Beclin-1, p62 and mTOR pathway in spinal cord neurons. (A) Expression of LC3-II, Beclin-1 and p62 in spinal cord neurons. (B) Semi-quantity analysis of LC3-II/ $\beta$-actin expression. (C) Semi-quantity analysis of Beclin-1/ $\beta$-actin expression. (D) Semi-quantity analysis of p62/ $\beta$-actin expression, ${ }^{*} \mathrm{P}<0.05,{ }^{* *} \mathrm{P}<0.01$, compared between groups, $\mathrm{n}=6$. (E) Expression of p-p70S6K, p70S6K, p-mTOR and mTOR in spinal cord neurons. (F) Semi-quantity analysis of p-p70S6K/p70S6K, p-mTOR/mTOR expression, ${ }^{*} \mathrm{P}<0.05$, ${ }^{* *} \mathrm{P}<0.01$, compared between groups, $\mathrm{n}=6$. LC3, light chain 3; mTOR, mechanistic target of rapamycin.

demonstrated as a main mechanism of spinal cord neuronal death induced by oxidative stress, the effect of pterostilbene on apoptosis was also investigated by TUNEL staining. The TUNEL staining showed that apoptosis incidence was induced by $10 \mu \mathrm{M} \mathrm{H}_{2} \mathrm{O}_{2}$; however pterostilbene significantly inhibited the apoptosis incidence in the $\mathrm{H}_{2} \mathrm{O}_{2}$-treated cells $(\mathrm{P}<0.05$, Fig. 6). ATG5 siRNA significantly increased the apoptosis incidence in the cells treated with pterostilbene combined with $\mathrm{H}_{2} \mathrm{O}_{2}(\mathrm{P}<0.05$, Fig. 6), suggesting that autophagy inhibiton reversed the inhibitory effect of pterostilbene on apoptosis.

\section{Discussion}

The secondary injuries after severe SCI are composed of ischemia-reperfusion injury, inflammation, edema, oxidative stress induced by ROS over-expression, glutamate-induced cytotoxicity, accumulation of intracellular calcium, cell apoptosis and necrosis (15). Glutamate accumulation in cells after SCI leads to the influx of intracellular calcium, and mitochondrial membrane permeability transition (MPT) induced by the absorption of intracellular calcium results in the over-production of ROS, leading to the oxidative stress in spinal cord neurons $(15,16)$. Oxidative stress contributes to the complex secondary injury cascades, therefore, antioxidants might be helpful to alleviate the SCI damage by maintaining the oxidative/antioxidative balance (15).

Resveratrol, a well-acknowledged anti-oxidant, can relieve the progression of various diseases, such as cardiovascular diseases and osteoarthritis, but the poor bioavailability of resveratrol impedes the clinical translation (17). By contrast, pterostilbene, a resveratrol analogue, has a smaller molecule and longer half-life with a relatively high bioavailability (5). Therefore, pterostilbene aroused more attention from researchers compared with resveratrol. Pterostilbene not only inhibited endothelial cell apoptosis induced by oxidative stress (18), but also activated autophagy of endothelial cells through the AMPK $\alpha 1-m T O R$ signaling pathway (12). In a variety of tumor cells, pterostilbene could also activate 
A

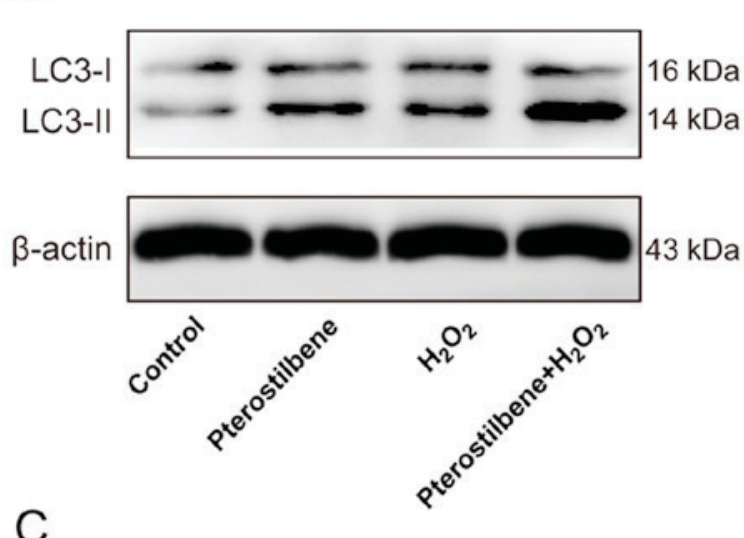

C

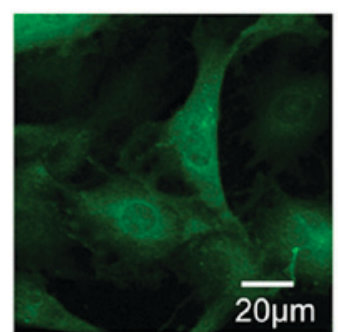

Control

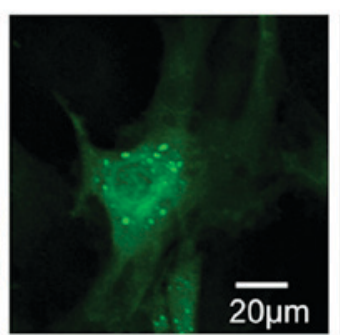

Pterostilbene

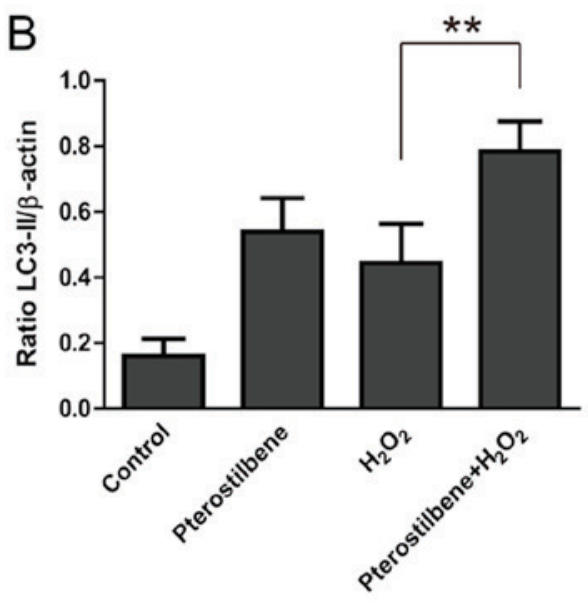

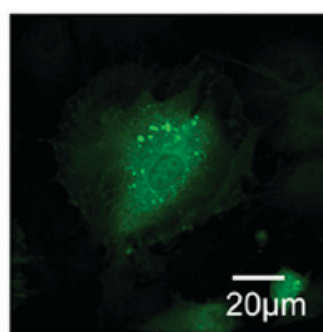

$\mathrm{H}_{2} \mathrm{O}_{2}$

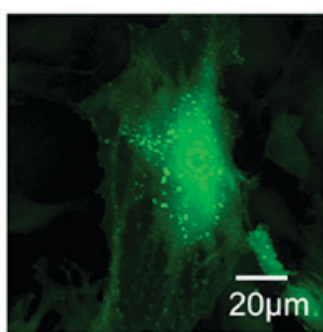

Pterostilbene+ $\mathrm{H}_{2} \mathrm{O}_{2}$

Figure 3. The effect of pterostilbene on the autophagic level in spinal cord neurons treated with $\mathrm{H}_{2} \mathrm{O}_{2}$. (A) Western blotting for LC3-II expression. (B) Semi-quantity analysis of LC3-II/ $\beta$-actin expression, ${ }^{* *} \mathrm{P}<0.01$, compared between groups, $\mathrm{n}=6$. (C) Autophagosomes in cells after transfection with GFP-C3 adenovirus under confocal microscopy. LC3, light chain 3; GFP, green fluorescent protein.

A

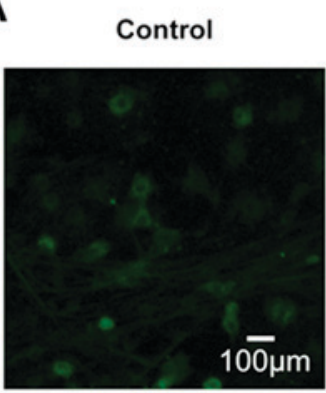

Pterostilbene

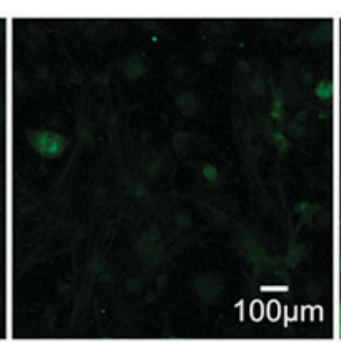

B

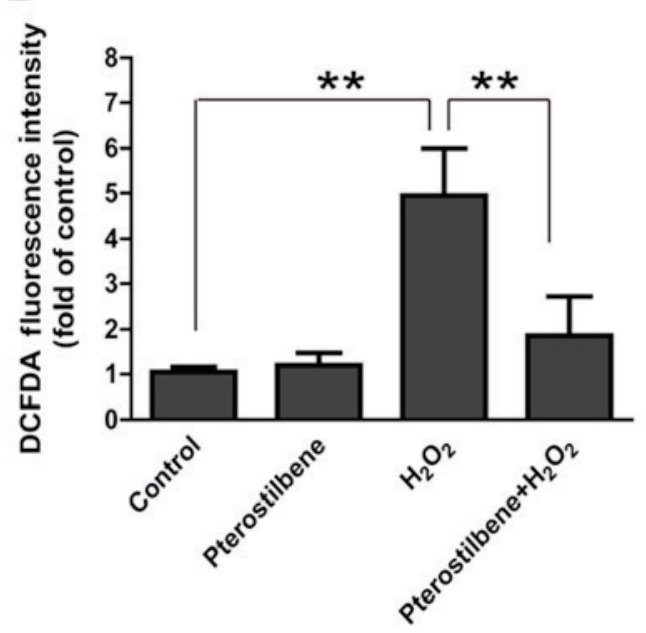

$\mathrm{H}_{2} \mathrm{O} 2$

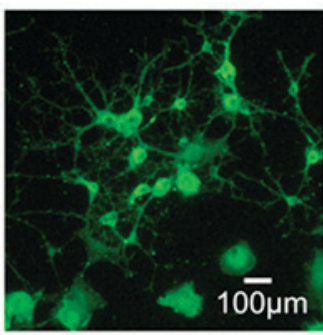

C

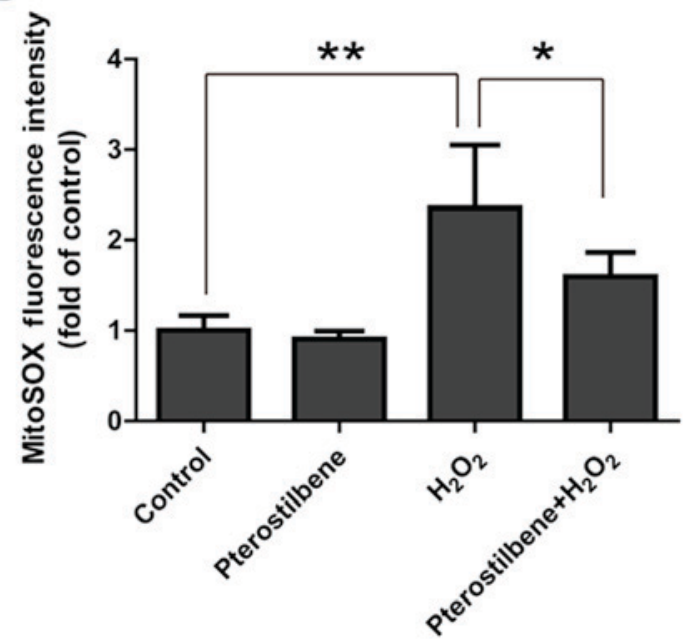

Figure 4. The effect of pterostilbene on ROS production in spinal cord neurons treated with $\mathrm{H}_{2} \mathrm{O}_{2}$. (A) DCFDA staining for primary spinal cord neurons.

(B) Quantity analysis of intensity of the DCFDA staining, ${ }^{* *} \mathrm{P}<0.01$, compared between groups. (C) Quantity analysis of intensity of the MitoSOx staining,

${ }^{* * *} \mathrm{P}<0.01$, compared between groups, $\mathrm{n}=6$. ROS, reactive oxygen species. 
A

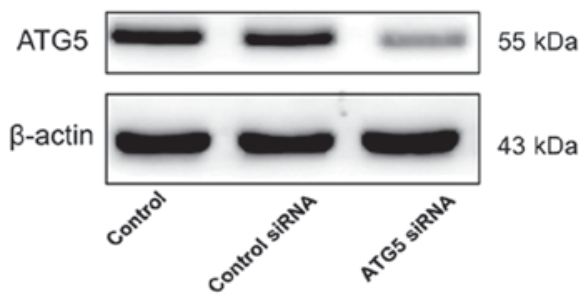

C

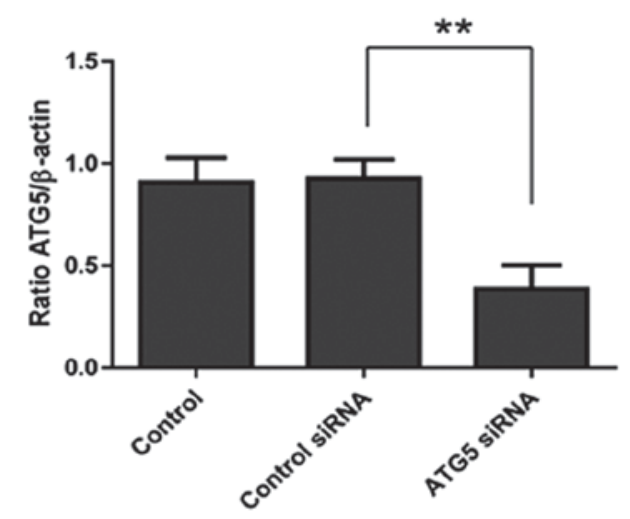

$E$

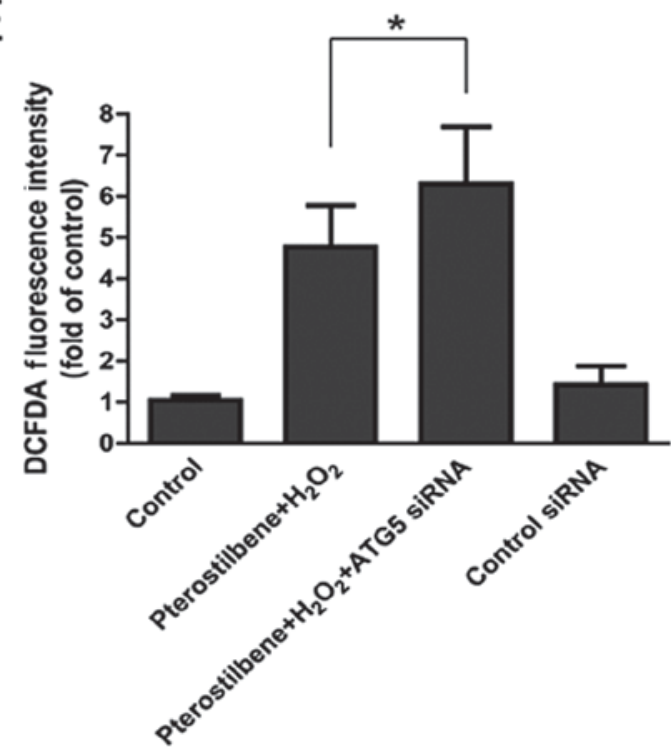

B
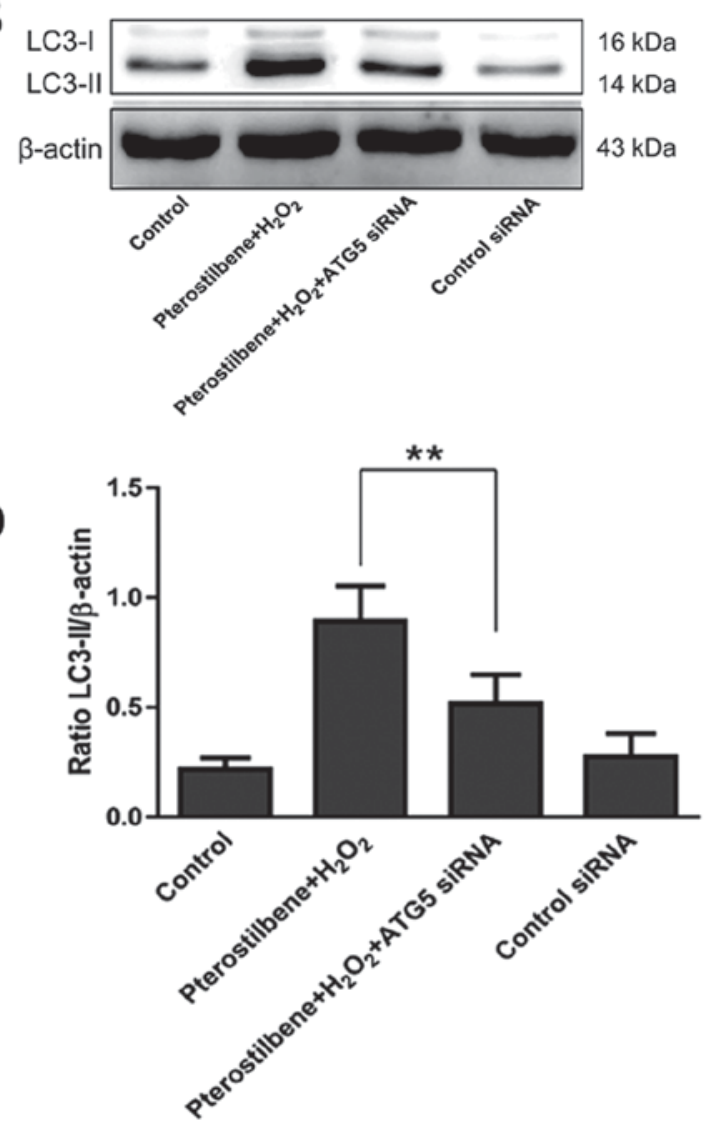

$\mathrm{F}$

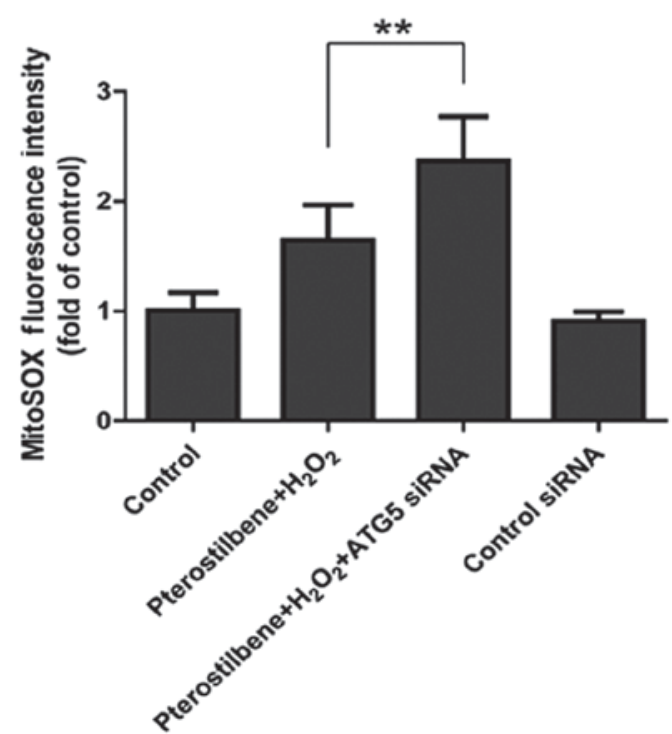

Figure 5. The effect of autophagic inhibition on the ROS production regulated by the pterostilbene in spinal cord neurons. (A) ATG5 expression after ATG5 siRNA transfection. (B) LC3-II expression after ATG5 silence. (C) Semi-quantity analysis of the ATG5/ $\beta$-actin expression, ${ }^{* *} \mathrm{P}<0.01$, compared with each group, $n=6$. (D) Semi-quantity analysis of LC3-II/ $\beta$-actin expression, ${ }^{* *} \mathrm{P}<0.01$, compared with each group, $\mathrm{n}=6$. (E) DCFDA staining after ATG5 siRNA, ${ }^{\prime} \mathrm{P}<0.05$, compared with each group, $\mathrm{n}=6$. (F) MitoSOX staining after ATG5 siRNA, ${ }^{* *} \mathrm{P}<0.01$, compared with each group, $\mathrm{n}=6$. ROS, reactive oxygen species; LC3, light chain 3.

autophagy and exert anticancer effect (13). Although pterostilbene has certain cytotoxicity to tumor cells, we first confirmed that less than $20 \mu \mathrm{M}$ pterostilben had no cytotoxic effect on primary spinal cord neurons, providing a basis for the following researches. In the paper, we found that pterostilbene activated autophagy in spinal cord neurons by inhibiting mTOR pathway, which was demonstrated by the LC3-I, Beclin-1 and p62 western blotting and GFP-LC3 assay. The promontory effect of pterostilbene on autophagy was further demonstrated in the $\mathrm{H}_{2} \mathrm{O}_{2}$-treated cells. In addition, pterostilbene inhibited the increase in ROS production induced by $\mathrm{H}_{2} \mathrm{O}_{2}$, and the inhibition of autophagy could reverse the protection, indicating the involvement of autophagy activation. Finally, the inhibitory effect of pterostilbene on apoptosis through autophagy 

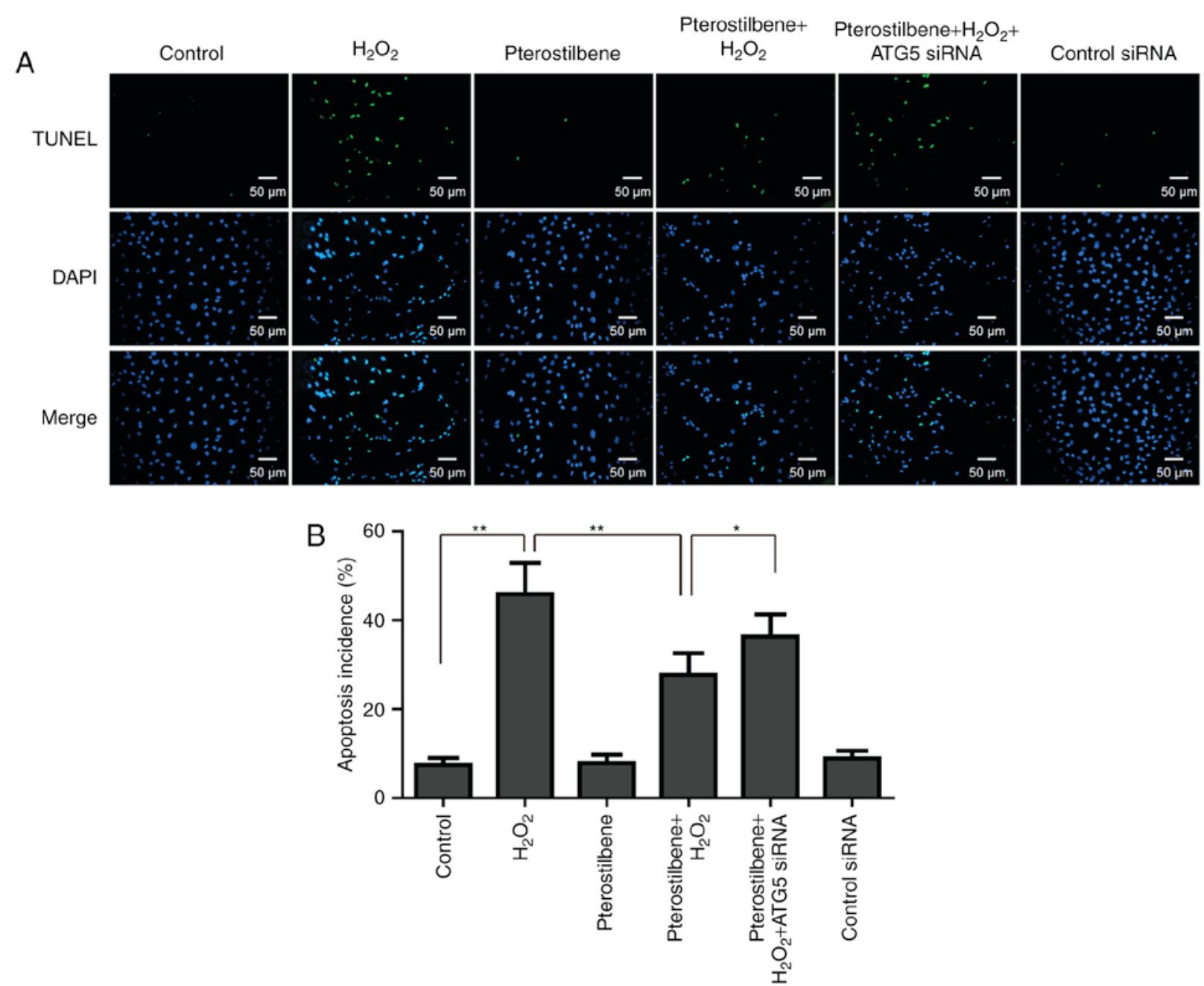

Figure 6. The effect of pterostilbene and autophagy on the apoptosis. (A) TUNEL analysis of primary spinal cord neurons. (B) Semi-quantity analysis of apoptosis percentage according to the TUNEL analysis, ${ }^{*} \mathrm{P}<0.05,{ }^{* * *} \mathrm{P}<0.01$, compared with each group, $\mathrm{n}=6$.

activation was also demonstrated by using the TUNEL assay. Taken together, these founds might provide a theoretical and experimental basis for future clinical treatment of SCI injury.

Spinal cord neuron is a cell type with high demanding of energy, therefore the number of mitochondria in the cytoplasm is larger than other cells in the body. In addition, energy generation depends on the mitochondria, leading to the mitochondrial ROS production in cells under oxidative stress (19). On the other hand, ROS production disturbs the energy synthesis in mitochondria and causes apoptosis and necrosis (20). In this paper, $\mathrm{H}_{2} \mathrm{O}_{2}$ was used to simulate the oxidative stress, and DCFDA and MitoSOX staining demonstrated the increase in ROS production induced by $\mathrm{H}_{2} \mathrm{O}_{2} \cdot \mathrm{H}_{2} \mathrm{O}_{2}$ causes the injury of mitochondria, nucleic acids and proteins via a Fenton reaction (20), which might be a mechanism of apoptosis under oxidative stress.

Oxidative stress is caused by an imbalance in production and clearance of intracellular ROS and active nitrogen. Recently, it has been reported that pterostilbene could inhibit the production of ROS in a variety of cells. In neuronal HT22 cells of mouse hippocampus, pterostilbene inhibits glutamate and high glucose-induced ROS by Nrf2 (NF-E2)-related factor 2 signaling pathway $(21,22)$. In this study, we validated that pterostilbene inhibited $\mathrm{H}_{2} \mathrm{O}_{2}$-induced ROS production and confirmed the role of pterostilbene in antioxidant stress in spinal cord neurons.

Recently, accumulating evidences have suggested that autophagy is an essential cellular antioxidant pathway. In neural stem cell, defect of autophagy resulted in a rise in ROS production (23). Furthermore, autophagy has been reported to regulate the nuclear translocation of Nrf2 which is a transcription factor of anti-oxidant genes (24). Therefore, Nrf2 might be a mechanism by which autphagy inhibits the ROS production. Given the relationship between autophagy and oxidative stress, autophagy might be a promising mechanism involved in the effect of pterostilbene on ROS production in spinal cord neurons. In this paper, autophagy inhibition by ATG5 silencing reversed the protection of pterostilbene against on ROS production, demonstrating the participation of autophagy in the regulation; however, the downstream of autophagy required further researches.

Cell apoptosis is also a major secondary pathological change of SCI. Autophagy and apoptosis have crosstalks in spinal cord neurons (25). Resveratrol could inhibit apoptosis and activate autophagy via SIRT1/AMPK signaling pathway after SCI in rats (25). Electroacupuncture preconditioning and postconditioning could reduce apoptosis induced by spinal cord ischemia reperfusion via activating autophagy (26). In the 
paper, autophagy inhibition by ATG5 silencing increased apoptosis perecentage detected by TUNEL assay, demonstrating the involvement of autophagy in the effect of pterostilbene on apoptosis.

In summary, $20 \mu \mathrm{M}$ pterostilbene is not cytotoxic to the primary spinal cord neurons and increases autophagy levels under normal and oxidative stress condotions. mTOR pathway was inhibited by pterostilbene. Pterostilbene inhibits ROS production and apoptosis induced by $\mathrm{H}_{2} \mathrm{O}_{2}$, but autophagy inhibition by ATG5 silencing reverses the protection of pterostilbene against ROS production and apoptosis in spinal cord neurons. Taken together, pterostilbene inhibits ROS production and apoptosis in spinal cord neurons by activating autophagy via mTOR pathway.

\section{References}

1. Chen HC, Fong TH, Lee AW and Chiu WT: Autophagy is activated in injured neurons and inhibited by methylprednisolone after experimental spinal cord injury. Spine (Phila Pa 1976) 37: 470-475, 2012

2. Wang JF, Li Y, Song JN and Pang HG: Role of hydrogen sulfide in secondary neuronal injury. Neurochem Int 64: 37-47, 2014.

3. Jia Z, Zhu H, Li J, Wang X, Misra H and Li Y: Oxidative stress in spinal cord injury and antioxidant-based intervention. Spinal Cord 50: 264-274, 2012.

4. Liu D, Liu J, Sun D and Wen J: The time course of hydroxyl radical formation following spinal cord injury: The possible role of the iron-catalyzed Haber-Weiss reaction. J Neurotrauma 21 805-816, 2004

5. Remsberg CM, Yáñez JA, Ohgami Y, Vega-Villa KR, Rimando AM and Davies NM: Pharmacometrics of pterostilbene: Preclinical pharmacokinetics and metabolism, anticancer, antiinflammatory, antioxidant and analgesic activity. Phytother Res 22: 169-179, 2008.

6. Li D, Song $\mathrm{T}$, Yang $\mathrm{L}$, Wang $\mathrm{X}$, Yang $\mathrm{C}$ and Jiang $\mathrm{Y}$ : Neuroprotective actions of pterostilbene on hypoxic-ischemic brain damage in neonatal rats through upregulation of heme oxygenase-1. Int J Dev Neurosci 54: 22-31, 2016.

7. Zhou Y, Zhang XM, Ma A, Zhang YL, Chen YY, Zhou H, Li WJ and Jin X: Orally administrated pterostilbene attenuates acute cerebral ischemia-reperfusion injury in a dose- and time-dependent manner in mice. Pharmacol Biochem Behav 135: 199-209, 2015.

8. Mizushima N, Levine B, Cuervo AM and Klionsky DJ: Autophagy fights disease through cellular self-digestion. Nature 451: 1069-1075, 2008

9. Jiang LB, Lee S, Wang Y, Xu QT, Meng DH and Zhang J: Adipose-derived stem cells induce autophagic activation and inhibit catabolic response to pro-inflammatory cytokines in rat chondrocytes. Osteoarthritis Cartilage 24: 1071-1081, 2016.

10. Jiang L, Yuan F, Yin X and Dong J: Responses and adaptations of intervertebral disc cells to microenvironmental stress: A possible central role of autophagy in the adaptive mechanism. Connect Tissue Res 55: 311-321, 2014

11. Su M, Guan H, Zhang F, Gao Y, Teng X and Yang W: HDAC6 regulates the chaperone-mediated autophagy to prevent oxidative damage in injured neurons after experimental spinal cord injury. Oxid Med Cell Longev 2016: 7263736, 2016.
12. Zhang L, Cui L, Zhou G, Jing H, Guo Y and Sun W: Pterostilbene, a natural small-molecular compound, promotes cytoprotective macroautophagy in vascular endothelial cells. J Nutr Biochem 24 . 903-911, 2013

13. Ko CP, Lin CW, Chen MK, Yang SF, Chiou HL and Hsieh MJ: Pterostilbene induce autophagy on human oral cancer cells through modulation of Akt and mitogen-activated protein kinase pathway. Oral Oncol 51: 593-601,2015.

14. Anderson KN, Potter AC, Piccenna LG, Quah AK, Davies KE and Cheema SS: Isolation and culture of motor neurons from the newborn mouse spinal cord. Brain Res Brain Res Protoc 12: 132-136, 2004.

15. Fatima G, Sharma VP, Das SK and Mahdi AA: Oxidative stress and antioxidative parameters in patients with spinal cord injury: Implications in the pathogenesis of disease. Spinal Cord 53: 3-6, 2015.

16. McEwen ML, Sullivan PG and Springer JE: Pretreatment with the cyclosporin derivative, NIM811, improves the function of synaptic mitochondria following spinal cord contusion in rats. J Neurotrauma 24: 613-624, 2007.

17. Subramanian L, Youssef S, Bhattacharya S, Kenealey J, Polans AS and van Ginkel PR: Resveratrol: Challenges in translation to the clinic-a critical discussion. Clin Cancer Res 16: 5942-5948, 2010.

18. Zhang L, Zhou G, Song W, Tan X, Guo Y, Zhou B, Jing H, Zhao S and Chen L: Pterostilbene protects vascular endothelial cells against oxidized low-density lipoprotein-induced apoptosis in vitro and in vivo. Apoptosis 17: 25-36, 2012.

19. Hall ED: Lipid peroxidation. Adv Neurol 71: 247-258, 1996.

20. Fariss MW, Chan CB, Patel M, Van Houten B and Orrenius S: Role of mitochondria in toxic oxidative stress. Mol Interv 5 : 94-111, 2005.

21. Wang B, Liu H, Yue L, Li X, Zhao L, Yang X, Wang X, Yang Y and Qu Y: Neuroprotective effects of pterostilbene against oxidative stress injury: Involvement of nuclear factor erythroid 2-related factor 2 pathway. Brain Res 1643: 70-79, 2016.

22. Yang Y, Fan C, Wang B, Ma Z, Wang D, Gong B, Di S, Jiang S, Li Y, Li T, et al: Pterostilbene attenuates high glucose-induced oxidative injury in hippocampal neuronal cells by activating nuclear factor erythroid 2-related factor 2. Biochim Biophys Acta 1863: 827-837, 2017

23. Wang C, Liang CC, Bian ZC, Zhu Y and Guan JL: FIP200 is required for maintenance and differentiation of postnatal neural stem cells. Nature Neurosci 16: 532-542, 2013.

24. Taguchi K, Fujikawa N, Komatsu M, Ishii T, Unno M, Akaike T, Motohashi $\mathrm{H}$ and Yamamoto M: Keapl degradation by autophagy for the maintenance of redox homeostasis. Proc Natl Acad Sci USA 109: 13561-13566, 2012.

25. Zhao H, Chen S, Gao K, Zhou Z, Wang C, Shen Z, Guo Y, Li Z, Wan Z, Liu C and Mei X: Resveratrol protects against spinal cord injury by activating autophagy and inhibiting apoptosis mediated by the SIRT1/AMPK signaling pathway. Neuroscience 348: 241-251, 2017.

26. Fang B, Qin M, Li Y, Li X, Tan W, Zhang Y and Ma H: Electroacupuncture preconditioning and postconditioning inhibit apoptosis and neuroinflammation induced by spinal cord ischemia reperfusion injury through enhancing autophagy in rats. Neurosci Lett 642: 136-141, 2017.

This work is licensed under a Creative Commons Attribution-NonCommercial-NoDerivatives 4.0 International (CC BY-NC-ND 4.0) License. 